\title{
Efeito de diferentes intensidades de exercício em esteira sobre os parâmetros eletrocardiográficos de cães hígidos
}

\author{
[Effect of different intensities of treadmill exercise on eletrocardiographic
} parameters of healthy dogs]

J.S. Xavier, J. Volpato, A.C. Dalmina, C.D.L. Cancelier, Á. Costa, E.L.C. Pereira, L. Serafini, M.E. Saito, L.A. Yonezawa*

Centro de Ciências Agroveterinárias (CAV), Universidade do Estado de Santa Catarina (UDESC) - Lages, SC

\section{RESUMO}

Objetivou-se a padronização de testes de exercício de alta e baixa intensidades em esteira, bem como a avaliação do eletrocardiograma de cães submetidos a esses testes. Para tal fim, sete cães da raça Australian Cattle Dog e quatro da raça Border Collie clinicamente saudáveis foram submetidos a dois testes de exercício em esteira, com pelo menos sete dias de intervalo: T1 - teste de exercício de alta intensidade e curta duração, e T2 - teste de exercício de baixa intensidade e longa duração. A amplitude e a duração de ondas e intervalos foram avaliadas no momento antes do exercício (M0) e nos momentos imediatamente após o término dos testes (MPE) e 30 minutos após (M30). A frequência e o ritmo cardíacos foram avaliados antes dos testes e continuamente por 30 minutos após o término do exercício. Verificou-se diferença significativa somente para a duração do intervalo QT em M30 em T1, além de algumas arritmias, como complexos atriais e ventriculares prematuros isolados em três animais após o teste T1, e em quatro após T2. Os testes de exercício foram adequados para promover estimulação simpática nos cães, contudo não causaram alterações significativas no eletrocardiograma, provavelmente em razão do excelente condicionamento físico dos animais.

Palavras-chave: cão, eletrocardiograma, fisiologia do exercício

\begin{abstract}
This study aimed to stardardize high and low intensity exercise tests, and evaluate the electrocardiogram of dogs submitted to these tests. Seven clinically healthy Australian Cattle dogs and four Border Collies underwent two exercise treadmill tests, with at least a seven day interval: T1 - high intensity and short duration exercise test, and T2 - low intensity and long duration exercise test. Amplitude and duration of waves and intervals were assessed at resting time before exercise (MO), at immediately after (MPE) and at 30 minutes (M30) after the end of the tests. Heart rate and cardiac rhythm were evaluated before the tests and continuously for 30 minutes after the end of exercise. There was a significant difference only for duration of the QT interval at M30 in T1, and some arrhythmias such as isolated atrial and ventricular premature complexes in three animals after T1 test, and in four dogs after T2. The exercise tests of the present study was suitable to promote sympathetic stimulation in dogs, however did not cause significant changes on the electrocardiogram probably because of the excellent physical fitness of the dogs.
\end{abstract}

Keywords: dog, electrocardiogram, exercise physiology

\section{INTRODUÇÃO}

Recentemente os estudos relacionados ao exercício físico em cães vêm aumentando cada vez mais para melhor compreensão de sua fisiologia, uma vez que esses animais

Recebido em 12 de setembro de 2016

Aceito em 30 de setembro de 2016

*Autor para correspondência (corresponding author)

E-mail: leticiay@gmail.com desempenham ampla variedade de esportes e de atividades físicas (Piccione et al., 2012; Barretto et al., 2013; Radin et al., 2015). Na região Serrana do estado de Santa Catarina, há um crescente número de cães da raça Australian Cattle Dog, também conhecida como Boiadeiro Australiano, e da raça Border Collie. Ambas as 
raças são muito eficientes para se utilizar no trabalho de pastoreio e, por isso, destacam-se nessa região, pois a agropecuária é muito importante para a economia local. Adicionalmente, são cães muito inteligentes, fortes e ágeis, e trabalham durante a maior parte do dia em fazendas, o que exige grande esforço físico (Briggs, 2001).

A prática de atividade física regular pode promover o desenvolvimento de diversos sistemas orgânicos, bem como a modulação do sistema nervoso autônomo, resultando em aumento do tônus vagal e diminuição do tônus simpático (Billman, 2009; Barretto et al., 2013). Durante o exercício, ocorrem diversas adaptações fisiológicas, sendo necessários ajustes orgânicos, principalmente cardiovasculares e respiratórios, para compensar e manter o esforço realizado (Ascensão et al., 2003). Contudo, a prática inadvertida de exercício físico pode interferir na modulação autonômica do coração, podendo até predispor o surgimento de arritmias (Barretto et al., 2013). Acredita-se que o exercício intenso induz o aumento do consumo de oxigênio pelo miocárdio e há produção excessiva de espécies reativas de oxigênio que podem contribuir para lesão de membranas celulares do miocárdio por lipoperoxidação (Ascensão et al., 2003). A hipóxia miocárdica e subsequente reperfusão estão relacionadas à produção desses radicais de oxigênio e podem culminar em instabilidade elétrica e aumento do potencial arritmogênico do músculo cardíaco (Leroux et al., 1995).

Diversas cardiopatias podem culminar em queda de desempenho e/ou intolerância ao exercício físico em cães, dentre as quais se destacam as anomalias congênitas, a endocardiose de valvas atrioventriculares e a cardiomiopatia dilatada (Taylor, 2011; Agudelo e Schanilec, 2013; Steinberg, 2013). Essas doenças cardíacas podem causar taqui ou bradiarritmias, que reduzem o débito cardíaco e a perfusão tecidual, e, em consequência, podem proporcionar fraqueza, síncope ou até mesmo a morte súbita devido à menor perfusão e hipóxia teciduais. $\mathrm{O}$ diagnóstico fica dificultado, uma vez que os achados clínicos de auscultação, pulso arterial, radiografias torácicas, eletrocardiograma e ecocardiografia podem se apresentar normais no repouso (Taylor, 2011).
O eletrocardiograma (ECG) é o exame de escolha para avaliação da atividade elétrica cardíaca e permite o diagnóstico das arritmias. Embora seja essencial para avaliação do ritmo, o ECG de repouso possui diversas limitações, visto que ocasionalmente podem ocorrer alterações de ritmo durante ou mesmo após atividade física, as quais não são manifestadas no repouso. Ainda, alguns animais podem apresentar arritmias consideradas fisiológicas durante o repouso, provocadas por tônus parassimpático elevado, que podem ser confundidas com arritmias devido à doença cardíaca. Dessa forma, para o reconhecimento dos tipos de arritmias, se são provocadas pelo exercício ou se são abolidas por ele, torna-se necessária a avaliação eletrocardiográfica durante ou após um exercício extenuante, quando o tônus simpático e o consumo de oxigênio estão aumentados e a influência parassimpática está reduzida (Sleeper, 2008; Yonezawa et al., 2014). Segundo Sleeper (2008), o ECG pós-exercício é indicado para cães com doenças cardíacas ocultas, ou seja, assintomáticas. Nesses animais, a combinação de exercício, doença miocárdica e hipertrofia de ventrículo pode resultar em indicadores de isquemia miocárdica no ECG, como depressão de segmento ST e ectopia ventricular. No entanto, a ausência de alterações não descarta cardiopatia.

Embora as atividades físicas a campo, como treinamento de cães ou competições, sejam de fácil realização, a padronização e a repetitividade desses tipos de exercício podem ser difíceis. Desse modo, a padronização de testes de exercício em esteira permite maior controle sobre a intensidade ou carga de trabalho, a duração do exercício e as condições climáticas do ambiente, favorecendo a repetitividade dos testes (Ferasin e Marcora, 2009). Além disso, é muito importante garantir a segurança dos animais durante $\mathrm{O}$ exercício na esteira. Atualmente, há diversos trabalhos reportando diferentes protocolos de exercício em esteira para cães (Ferasin e Marcora, 2009; Piccione et al., 2012; Marcondes-Santos et al., 2015; Radin et al., 2015). O exercício padronizado em esteira também é amplamente recomendado para pacientes obesos que estão em programas de perda de peso (Chauvet et al., 2011; Vitger et al., 2016) e para cardiopatas, a fim de melhorar a qualidade de vida e a tolerância ao exercício (Marcondes-Santos et al., 2015). 
O presente estudo teve como objetivos a padronização dos testes de exercício de alta e baixa intensidades em esteira, bem como a avaliação de possíveis alterações eletrocardiográficas causadas pelos testes em cães clinicamente saudáveis das raças Australian Cattle Dog e Border Collie.

\section{MATERIAL E MÉTODOS}

O projeto de pesquisa foi avaliado e aprovado pelo Comitê de Ética em Experimentação Animal (Cetea) da Universidade do Estado de Santa Catarina (UDESC), sob protocolo $\mathrm{n}^{\circ}$ 01.03.14.

Para o estudo, foram selecionados 35 cães da raça Australian Cattle Dog, porém foram utilizados sete cães da raça Australian Cattle Dog e quatro da raça Border Collie, sendo seis machos e cinco fêmeas, com idade média de $2,4 \pm 2,0$ anos, peso médio de $19,8 \pm 3,9 \mathrm{~kg}$ e condição corporal normal. Os cães eram provenientes de proprietários do município de Lages/SC, os quais assinaram termo de consentimento para a realização deste estudo. Inicialmente, foi obtido o histórico dos animais, e estes foram avaliados por meio de exame físico completo (Feitosa, 2008), bem como eletrocardiograma de repouso (Tilley, 1992), ecocardiografia (Madron, 2015), hemograma (Jain, 1993) e bioquímica sérica (ureia, creatinina, alanina aminotransferase, fosfatase alcalina, aspartato aminotransferase, creatinaquinase, proteína, albumina, glicose e lactato), sendo considerados hígidos.

Todo o delineamento experimental foi realizado em salão com temperatura ambiente controlada por ar-condicionado digital. Nos momentos pré e pós-exercício, foram mensuradas a temperatura ambiente e a umidade relativa do ar por meio de termo-higrômetro digital.

Os cães foram submetidos a um primeiro teste de exercício físico (T1), com o protocolo de exercício de alta intensidade, que consistiu de cinco minutos na velocidade de $3,5 \mathrm{~km} / \mathrm{h}$ (passo) e cinco minutos na velocidade de $7,0 \mathrm{~km} / \mathrm{h}$ (trote), seguido do período de desaquecimento de cinco minutos na velocidade de $3,5 \mathrm{~km} / \mathrm{h}$, com 0\% de inclinação da esteira (Piccione et al., 2012 modificado). Após pelo menos sete dias de T1, foi realizado o segundo teste de exercício físico
(T2), cujo protocolo consistiu de 40 minutos na velocidade de $3,5 \mathrm{~km} / \mathrm{h}$ (passo), com $0 \%$ de inclinação.

Os parâmetros eletrocardiográficos e a temperatura retal foram avaliados no momento antes do exercício (M0) e nos momentos imediatamente após o término dos testes (MPE) e 30 minutos após (M30). Adicionalmente, foi realizada a avaliação da frequência cardíaca (FC) e do ritmo cardíaco antes dos testes e continuamente durante 30 minutos após o término dos testes de exercício.

Para a realização do exame eletrocardiográfico, foi utilizado um eletrocardiógrafo computadorizado (ECG PC Veterinário, TEB, Brasil). Os eletrodos foram posicionados conforme estabelecido por Tilley (1992), segundo o plano frontal de derivações bipolares e unipolares aumentadas. Devido à polipneia após os testes de exercício, os traçados eletrocardiográficos foram obtidos com os animais em posição quadrupedal para avaliação da frequência cardíaca, do ritmo e mensuração da amplitude e duração de ondas e intervalos nos momentos supracitados.

As análises foram processadas com o auxílio do programa estatístico computadorizado. De acordo com a distribuição normal dos dados, analisados pelo teste de Kolmogorov-Smirnov, as variáveis foram avaliadas segundo métodos estatísticos paramétricos, os quais inicialmente foram avaliados por meio do teste de análise de variância (ANOVA) de uma via para medidas repetidas e, quando o resultado mostrava-se significativo, as médias foram confrontadas pelo teste de comparação múltipla de médias de Tukey. Todas as análises foram consideradas significativas quando $\mathrm{P}<0,05$.

\section{RESULTADOS E DISCUSSÃO}

Neste estudo, a principal limitação foi o pequeno número de animais. Dos 35 cães da raça Australian Cattle Dog somente sete conseguiram executar ambos os testes de exercício. Piccione et al. (2012) relataram, em seu estudo com cães da raça Beagle, que todos os sete animais não eram pré-treinados ou familiarizados com a esteira e, mesmo assim, executaram o teste de 45 minutos. Cães com insuficiência cardíaca congestiva não familiarizados com a esteira 
também realizaram teste de exercício sem relutância, segundo Ferasin e Marcora (2007). Contudo, Ferasin e Marcora (2009) também tiveram dificuldades com alguns cães da raça Labrador, que se mostraram relutantes a caminhar na esteira. A maioria dos cães Australian Cattle Dog são criados soltos exatamente para condução do gado e, por esse motivo, houve muita dificuldade em submetê-los ao exercício em esteira, mesmo com diversas tentativas de condicionamento, como proposto por Kregel et al. (2006). Desse modo, optou-se pela inclusão de cães da raça Border Collie no estudo, uma vez que são utilizados para o mesmo tipo de trabalho. Todos os animais que conseguiram caminhar na esteira executaram os testes de exercício com facilidade. Além disso, estatisticamente não houve diferença entre as raças para os parâmetros avaliados. Os valores obtidos do eletrocardiograma, de temperatura retal e das condições do ambiente de ambos os testes estão apresentados nas Tab. 1 e 2.

Tabela 1. Média \pm desvio-padrão dos parâmetros eletrocardiográficos e de temperatura retal dos sete cães Australian Cattle Dog e quatro Border Collie submetidos a teste de exercício de alta intensidade (T1), nos momentos antes do exercício (M0), imediatamente após (MPE) e 30 minutos após o término do teste (M30), bem como dos parâmetros do ambiente (temperatura e umidade relativa do ar)

\begin{tabular}{ccccc}
\hline Parâmetro & M0 & MPE & M30 & P \\
\hline Frequência cardíaca (bpm) & $108,0 \pm 17,0$ & $122,0 \pm 16,0$ & $105,5 \pm 22,1$ & 0,096 \\
Duração de P (s) & $0,044 \pm 0,010$ & $0,040 \pm 0,004$ & $0,044 \pm 0,009$ & 0,585 \\
Amplitude de P (mV) & $0,235 \pm 0,053$ & $0,279 \pm 0,086$ & $0,240 \pm 0,099$ & 0,390 \\
Intervalo PR (s) & $0,104 \pm 0,018$ & $0,105 \pm 0,019$ & $0,104 \pm 0,023$ & 0,928 \\
Duração de QRS (s) & $0,052 \pm 0,010$ & $0,052 \pm 0,09$ & $0,052 \pm 0,08$ & 0,992 \\
Amplitude de QRS (mV) & $1,159 \pm 0,450$ & $1,200 \pm 0,408$ & $1,040 \pm 0,402$ & 0,655 \\
Intervalo QT (s) & $0,209 \pm 0,012 \mathrm{a}$ & $0,198 \pm 0,010 \mathrm{a}$ & $0,224 \pm 0,016 \mathrm{~b}$ & $<0,001$ \\
& & & & \\
Temperatura retal $\left({ }^{\circ} \mathrm{C}\right)$ & $38,8 \pm 0,5 \mathrm{a}$ & $39,4 \pm 0,4 \mathrm{~b}$ & $38,6 \pm 0,2 \mathrm{a}$ & $<0,001$ \\
& & & & \\
Temperatura ambiente $\left({ }^{\circ} \mathrm{C}\right)$ & $19,5 \pm 2,0$ & - & $19,2 \pm 1,5$ & 0,737 \\
Umidade relativa do ar $(\%)$ & $75,0 \pm 8,7$ & - & $80,5 \pm 7,8$ & 0,104
\end{tabular}

Letras minúsculas diferentes na mesma linha indicam diferença significativa entre momentos (teste de Tukey, $\mathrm{P}<0,05)$.

bpm: batimento por minuto; s: segundo; $\mathrm{mV}$ : milivolt; ${ }^{\circ} \mathrm{C}$ : grau Celsius.

O posicionamento padrão para se obter o traçado eletrocardiográfico é o decúbito lateral direito. Entretanto, neste estudo, o traçado foi obtido com o animal em posição quadrupedal. Tilley (1992) afirma que a posição quadrupedal pode ser utilizada para a análise de ritmo e dos intervalos, sendo especialmente útil nos animais com angústia respiratória e quando a contenção física possa ser prejudicial. Embora os animais não apresentassem angústia respiratória, após os testes de exercício foi constatada intensa polipneia e arquejo intenso e, por isso, optou-se pela posição quadrupedal, em acordo com Rovira et al. (2010), também na tentativa de minimizar os artefatos provocados pelos movimentos respiratórios. Desse modo, os valores de amplitude e a morfologia das ondas não são muito confiáveis.
Todos os valores de frequência cardíaca (FC), nos diferentes momentos e em ambos os testes, permaneceram dentro do limite de normalidade de 70 a 160bpm (Tilley e Smith Jr., 2008). Não houve diferença significativa dos valores da frequência cardíaca (FC) nos momentos M0, MPE e M30 em ambos os testes de exercício. Piccione et al. (2012) verificaram aumento significante da FC em cães da raça Beagle durante exercício em esteira, e Rovira et al. (2010) em cães submetidos à competição de agility, sendo esse aumento justificado pelo maior requerimento de oxigênio durante $o$ exercício. No presente estudo, não foi possível a mensuração da FC durante o exercício, mas provavelmente houve esse aumento em resposta ao estímulo simpático para aumentar o débito cardíaco. Contudo, verificou-se uma tendência de aumento da FC pós-exercício e de rápida redução para os valores pré-exercício durante a 
recuperação após o término dos testes (Fig. 1 e $2)$, mas sem diferença significativa $(\mathrm{P}=0,100$ para $\mathrm{T} 1, \quad \mathrm{P}=0,411$ para $\mathrm{T} 2)$. Apesar do rápido posicionamento dos eletrodos do eletrocardiógrafo após o término dos testes, um pequeno atraso de 30 segundos já é suficiente para alterar os resultados obtidos (Sleeper, 2008). Segundo Rovira et al. (2010), fatores como intensidade e duração do exercício, estado hidroeletrolítico, desempenho atlético, nível de treinamento e doenças subclínicas podem influenciar na resposta da FC ao exercício. A rápida recuperação da $\mathrm{FC}$ após o exercício pode ser justificada pelo fato de os cães serem muito bem condicionados fisicamente, de modo que os testes de exercício não apresentaram carga de trabalho suficiente para provocar grandes alterações nesses animais.

Tabela 2. Média \pm desvio-padrão dos parâmetros eletrocardiográficos e de temperatura retal dos sete cães Australian Cattle Dog e quatro Border Collie submetidos a teste de baixa intensidade (T2), nos momentos antes do exercício (M0), imediatamente após (MPE) e 30 minutos após o término do teste (M30), bem como dos parâmetros do ambiente (temperatura e umidade relativa do ar)

\begin{tabular}{ccccc}
\hline Parâmetro & M0 & MPE & M30 & P \\
\hline Frequência cardíaca (bpm) & $106,5 \pm 19,1$ & $116,0 \pm 17,4$ & $99,6 \pm 15,8$ & 0,105 \\
Duração de P (s) & $0,044 \pm 0,010$ & $0,041 \pm 0,003$ & $0,043 \pm 0,006$ & 0,776 \\
Amplitude de P (mV) & $0,230 \pm 0,050$ & $0,254 \pm 0,052$ & $0,254 \pm 0,048$ & 0,453 \\
Intervalo PR (s) & $0,104 \pm 0,016$ & $0,96 \pm 0,013$ & $0,098 \pm 0,013$ & 0,523 \\
Duração de QRS (s) & $0,054 \pm 0,008$ & $0,048 \pm 0,009$ & $0,054 \pm 0,007$ & 0,220 \\
Amplitude de QRS (mV) & $1,078 \pm 0,446$ & $1,038 \pm 0,269$ & $1,150 \pm 0,437$ & 0,796 \\
Intervalo QT (s) & $0,212 \pm 0,016$ & $0,196 \pm 0,014$ & $0,214 \pm 0,019$ & 0,070 \\
& & & & \\
Temperatura retal $\left({ }^{\circ} \mathrm{C}\right)$ & $38,6 \pm 0,3 \mathrm{a}$ & $39,0 \pm 0,2 \mathrm{~b}$ & $38,4 \pm 0,2 \mathrm{a}$ & $<0,001$ \\
& & & & $18,5 \pm 0,7$ \\
Temperatura ambiente $\left({ }^{\circ} \mathrm{C}\right)$ & $18,3 \pm 1,7$ & - & $76,3 \pm 7,4$ & 0,489 \\
Umidade relativa do ar $(\%)$ & $75,5 \pm 5,5$ & - & 0,792 \\
\hline
\end{tabular}

Letras minúsculas diferentes na mesma linha indicam diferença significativa entre momentos (teste de Tukey, $\mathrm{P}<0,05$ ).

bpm: batimento por minuto; s: segundo; $\mathrm{mV}$ : milivolt; ${ }^{\circ} \mathrm{C}$ : grau Celsius.

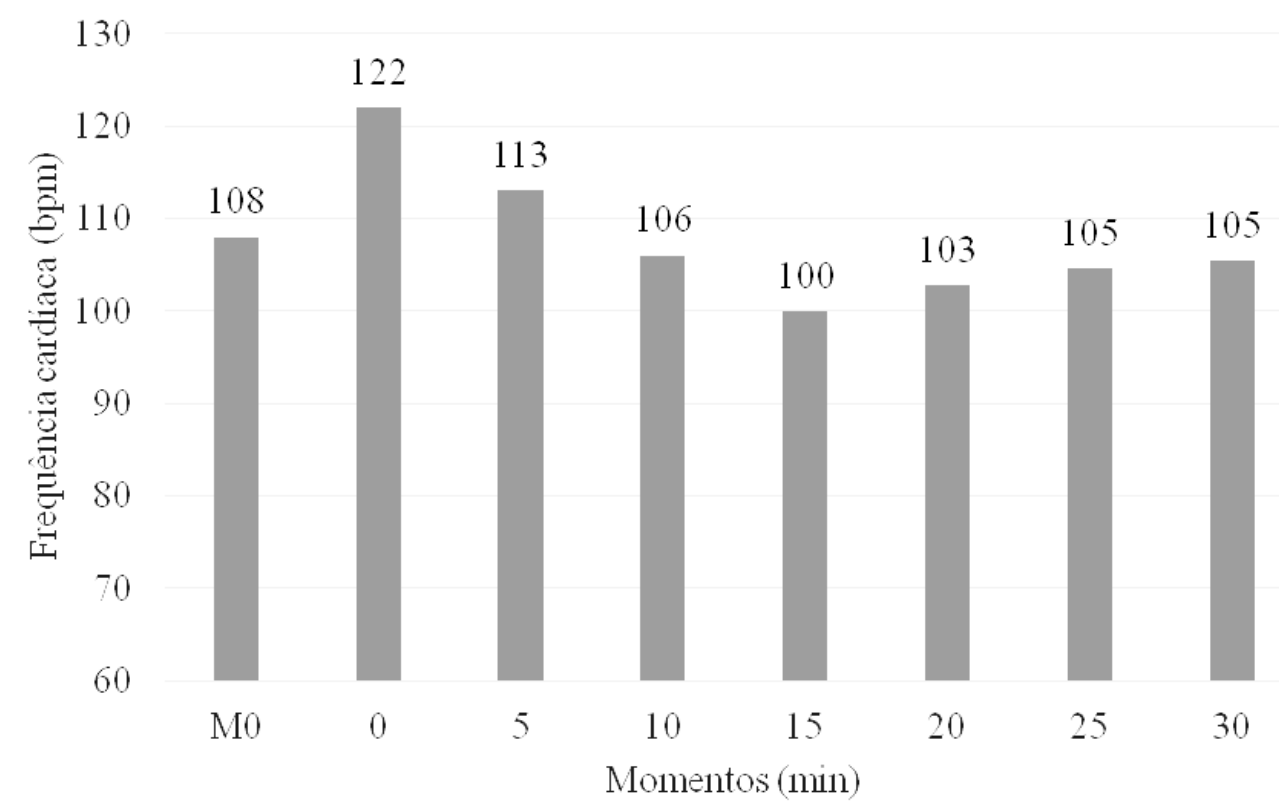

Figura 1. Variação da frequência cardíaca de 11 cães submetidos ao teste de alta intensidade (T1) nos momentos pré-exercício (M0) e após o término dos testes em diferentes momentos (0 a 30 min após). Teste de Tukey, $\mathrm{P}=0,100$. 


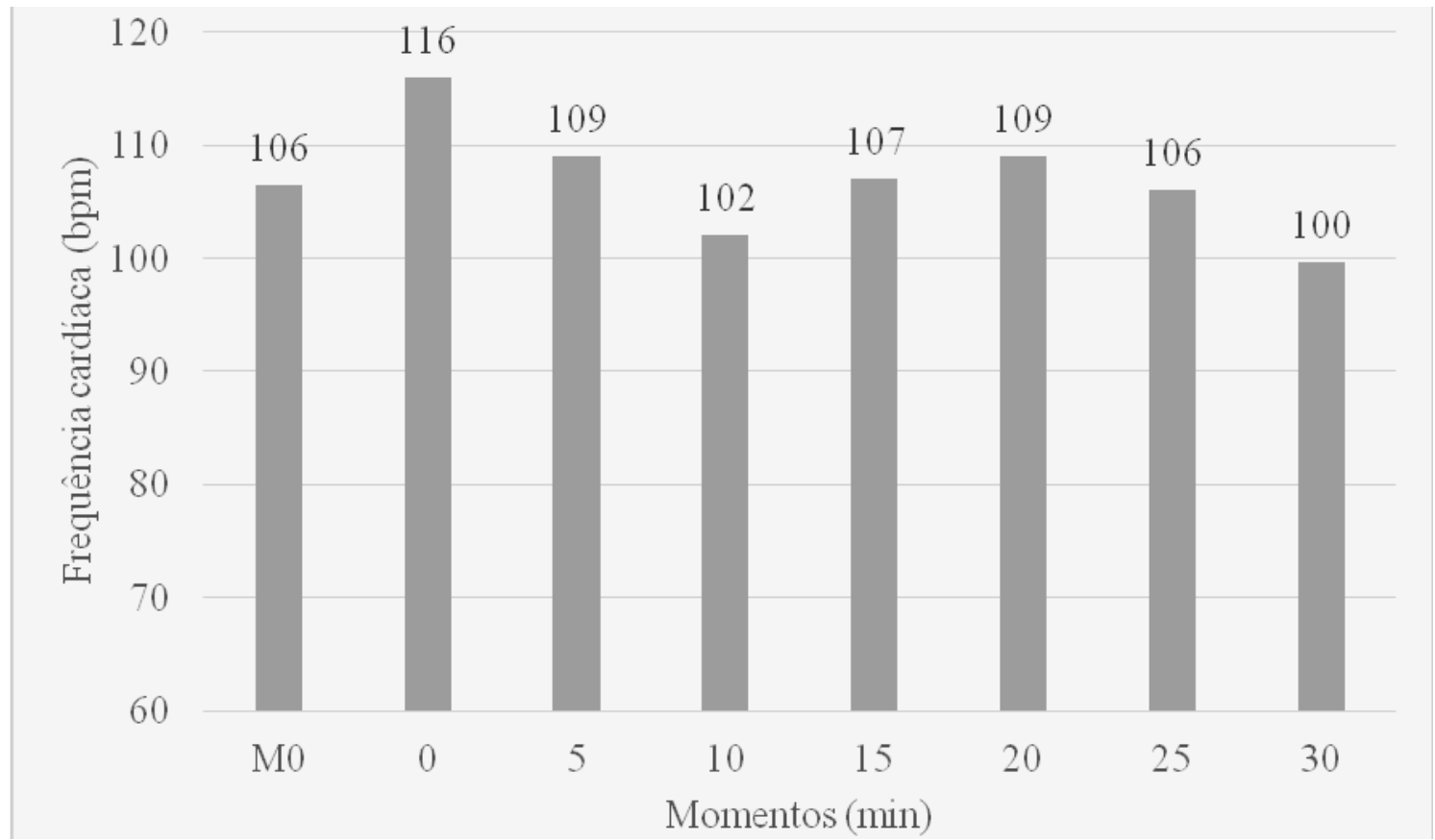

Figura 2. Variação da frequência cardíaca de 11 cães submetidos ao teste de baixa intensidade (T2) nos momentos pré-exercício (M0) e após o término dos testes em diferentes momentos (0 a 30 min após). Teste de Tukey, $\mathrm{P}=0,490$.

Os demais parâmetros eletrocardiográficos também permaneceram dentro do limite de referência (Tilley e Smith Jr., 2008) e não apresentaram diferença significativa, com exceção do intervalo QT. Foi observada uma discreta redução do intervalo QT imediatamente após o exercício em $\mathrm{T} 1$, com posterior aumento significativo em 30 minutos após o exercício. Esse fato pode ser explicado pela tendência à diminuição de QT em frequências aumentadas e pelo aumento de QT quando há menor FC (Tilley e Smith Jr., 2008), como também verificado por Yonezawa et al. (2009) em cavalos submetidos a exercício.

No momento basal, todos os animais avaliados apresentaram arritmia sinusal, e dois dos animais também apresentaram parada sinusal intermitente. Rovira et al. (2010) também verificaram arritmia sinusal antes do exercício em cães treinados e submetidos à competição de agility. A arritmia sinusal é considerada normal em cães, uma vez que a irregularidade é secundária às flutuações no tônus vagal associadas com o ciclo respiratório (Tilley e Smith Jr., 2008). A parada sinusal intermitente pode ser interpretada como uma arritmia sinusal "exagerada" (Tilley, 1992), sendo normal em cães com a variação do tônus vagal e sem sinais clínicos como síncope.

Imediatamente após o teste de exercício de alta intensidade (MPE-T1), 72,7\% (8/11) dos animais apresentaram arritmia sinusal e $27,3 \%$ (3/11) apresentaram ritmo sinusal normal. No MPE do teste de baixa intensidade $\mathrm{T} 2$, verificou-se que $81,8 \%$ (9/11) apresentaram arritmia sinusal e somente $18,2 \% \quad(2 / 11)$ apresentaram ritmo sinusal normal. Durante ou mesmo logo após uma atividade física, é esperada alteração do ritmo cardíaco em consequência do aumento da FC, que se eleva rapidamente no início do exercício e se estabiliza em dois a três minutos devido ao aumento do tônus simpático e à liberação de catecolaminas na circulação. A recuperação da FC ao seu valor normal é usualmente bem rápida no primeiro minuto após o exercício e depois decresce mais gradualmente até atingir os valores normais de repouso (Tilley e Smith Jr., 2008), o que também influencia no ritmo cardíaco. Barretto et al. (2013) verificaram episódios de taquicardia sinusal nos momentos de exercício em cães submetidos a diferentes atividades físicas e monitorados por sistema Holter, como resposta do organismo em atender a maior demanda de oxigênio. Como já discutido 
anteriormente, neste estudo não se verificou nem o aumento significativo da FC nem tampouco houve taquicardia sinusal nos cães após os testes de esforço, uma vez que os valores de FC não ultrapassaram o limite de referência. Entretanto, foram verificados em alguns animais $o$ desaparecimento da arritmia sinusal (M0) e o aparecimento do ritmo sinusal normal em MPE, o que pode ser indicativo de aumento da atividade simpática e de diminuição do tônus vagal. Os animais que apresentaram ritmo sinusal normal retornaram à condição de arritmia sinusal dentro de cinco minutos após o término dos testes, de modo que todos os animais apresentaram arritmia sinusal no M30 em ambos os testes.

Barretto et al. (2013) verificaram a ocorrência maior de arritmias, como os complexos atriais ou ventriculares prematuros, no grupo de cães saudáveis submetidos a atividades físicas e avaliados por Holter quando comparado ao grupo controle, estando algumas das arritmias relacionadas ao período durante ou após o exercício. Os autores não informaram se os cães apresentavam algum grau de condicionamento físico e concluíram que a prática inadvertida de exercícios físicos pode predispor a arritmias importantes. Já Rovira et al. (2010) não observaram nenhuma arritmia patológica em cães treinados submetidos ao exercício de agility. No presente estudo, verificou-se o aparecimento de complexos atriais e ventriculares prematuros isolados em alguns cães após os testes T1 e T2, conforme descrito na Tab. 3. Não foi possível atribuir nenhuma causa específica para essas arritmias, as quais parecem não ter significado clínico, como verificado em equinos pósexercício (Yonezawa et al., 2009). Como o exercício promove aumento do consumo de oxigênio pelo miocárdio, sugere-se que a produção acrescida de espécies reativas de oxigênio pode favorecer instabilidade elétrica e elevar o potencial arritmogênico cardíaco (Ascensão et al., 2003; Leroux et al., 1995). Acredita-se ainda que, pelo excelente condicionamento físico dos animais, o maior tônus parassimpático e o menor tônus simpático podem propiciar meios efetivos para reduzir a incidência de arritmias, principalmente as ventriculares (Billman, 2009).

Tabela 3. Arritmias observadas em quatro cães Australian Cattle Dog (A) e dois Border Collie (B) no período de até 30 minutos de recuperação pós-exercício dos testes de exercício de alta (T1) e baixa intensidades (T2)

\begin{tabular}{ccc}
\hline Animal & T1 & T2 \\
\hline $1 \mathrm{~A}$ & $1 \mathrm{VPC}$ & $2 \mathrm{VPC}$ \\
$5 \mathrm{~A}$ & - & $3 \mathrm{VPC}$ \\
$6 \mathrm{~A}$ & - & $1 \mathrm{APC}$ \\
$7 \mathrm{~A}$ & $1 \mathrm{APC}$ e & - \\
$10 \mathrm{~B}$ & $\mathrm{VPC}$ & - \\
$11 \mathrm{~B}$ & $1 \mathrm{APC}$ & $1 \mathrm{VPC}$ \\
\hline VPC: complexo & - & ventricular \\
complexo atrial prematuro.
\end{tabular}

O aumento significativo da temperatura retal dos cães foi verificado em MPE, o qual retornou próximo ao valor basal em M30, em ambos os testes. Como não houve alteração significativa da temperatura ambiente no salão, a elevação da temperatura retal dos animais foi ocasionada exclusivamente pela maior produção de calor da musculatura esquelética durante o exercício, que excedeu a capacidade do cão em dissipá-lo, em acordo com Piccione et al. (2012). Essa hipertermia transitória ocorre em consequência do aumento da intensidade do exercício e da atividade metabólica no músculo esquelético (Valberg, 2014). Dessa forma, a manutenção da temperatura ambiente controlada é importante para garantir conforto térmico dos animais durante o exercício e prevenir a fadiga muscular, uma vez que ambientes muito quentes e úmidos podem contribuir para aumento excessivo da temperatura do músculo esquelético, sendo um dos fatores que propiciam a fadiga (Valberg, 2014).

\section{CONCLUSÕES}

Concluiu-se que os testes de exercício padronizados neste estudo foram adequados para promover estimulação simpática nos cães das raças Australian Cattle Dog e Border Collie, mas não causaram alterações eletrocardiográficas significativas. 


\section{REFERÊNCIAS}

AGUDELO, C.F.; SCHANILEC, P. Evaluation of the functional capacity in dogs with naturally acquired heart disease. Vet. Med., v.58, p.264$270,2013$.

ASCENSÃO, A.; MAGALHÃES, J.; SOARES, J. et al. Exercício e stress oxidativo cardíaco. Rev. Port. Cardiol., v.22, p.651-678, 2003.

BARRETTO, F.L.; FERREIRA, F.S.; FREITAS, M.V. et al. Eletrocardiografia contínua (Holter) em cães saudáveis submetidos a diferentes exercícios físicos. Arq. Bras. Med. Vet. Zootec., v.65, p.1625-1634, 2013.

BILLMAN, G.E. Cardiac autonomic neural remodeling and susceptibility to sudden cardiac death: effect of endurance exercise training. Am. J. Physiol. Heart Circ. Physiol., v.297, p.H1171H1193, 2009.

BRIGGS, P.O. Livro gigante do cão. Lisboa: Centralivros, 2001. 416p

CHAUVET, A.; LACLAIR, J.; ELLIOTT, D.A.; GERMAN, A.J. Incorporation of exercise, using an underwater treadmill, and active client education into a weight management program for obese dogs. Can. Vet. J., v.52, p.491-496, 2011.

FEITOSA, F.L.F. Semiologia veterinária: a arte do diagnóstico. São Paulo: Roca, 2008. 735p.

FERASIN, L.; MARCORA, S. A pilot study to assess the feasibility of a submaximal exercise test to measure individual response to cardiac medication in dogs with acquired heart failure. Vet. Res. Commun., v.31, p.725-737, 2007.

FERASIN, L.; MARCORA, S. Reliability of an incremental exercise test to evaluate acute blood lactate, heart rate and body temperature responses in Labrador retrievers. J. Comp. Physiol. B, v.179, p.839-845, 2009.

JAIN, N.C. Essentials of veterinary hematology. Philadelphia: Lea \& Febiger, 1993. 417p.

KREGEL, K.C.; ALLEN, D.L.; BOOTH, F.W. et al. Exercise protocols using large animals: treadmill exercise in dogs. In: KREGEL, K.C.; ALLEN, D.L.; BOOTH, F.W. et al. Resource book for the design of animal exercise protocols. Am. Physiol. Soc., 2006. p.71-74.
LEROUX, A.J.; SCHOTT II, H.C.; HINES, M.T. Ventricular tachycardia associated with exhaustive exercise in a horse. J. Am. Vet. Med. Assoc., v.207, p.335-337, 1995.

MADRON, E. Normal views: 2D, TM, sprectral, and color Doppler. In: MADRON, E.; CHETBOUL, V.; BUSSADORI, C. Clinical echocardiography of the dog and cat. 2.ed. St. Louis: Elsevier.2015. p.3-19.

MARCONDES-SANTOS, M.; MANSUR, A.P.; FRAGATA, F.S.; STRUNZ, C.M.C. Short-term follow-up of exercise training program and betablocker treatment on quality of life in dogs with naturally acquired chronic mitral valve disease. Braz. J. Med. Biol. Res., v.48, p.886-894, 2015.

PICCIONE, G.; CASELLA, S.; PANZERA, M. et al. Effect of moderate treadmill exercise on some physiological parameters in untrained Beagle dogs. Exp. Anim., v.61, p.511-515, 2012.

RADIN, L.; BELIĆ, M.; BRKLJAČA BOTTEGARO, N. et al. Heart rate deflection point during incremental test in competitive agility border collies. Vet. Res. Commun., v.39, p.137-142, 2015.

ROVIRA, S.; MUÑOZ, A.; RIBER, C.; BENITO M. Heart rate, electrocardiographic parameters and arrhythmias during agility exercises in trained dogs. Rev. Méd. Vét., v.161, p.307-313, 2010.

SLEEPER, M.M. Special diagnostic techniques for evaluation of cardiac disease. In: TILLEY, L.P.; SMITH JR, F.W.K.; OYAMA, M.A.; SLEEPER, M.M. Manual of canine and feline cardiology. 4.ed. Philadelphia: WB Saunders, 2008. 443p.

STEINBERG, H.S. Common medical disorders that can affect performance. In: ZINK, M.C.; VAN DYKE, J.B. Canine sports medicine and rehabilitation. Hoboken: Willey-Blackwell, 2013. p.338-348.

TAYLOR, S.M. Diagnostic approach exercise intolerance in hunting dogs. In: EUROPEAN VETERINARY CONFERENCE, 46., 2011, Barcelona. Proceedings... Barcelona: AVEPA, 2011. In: <www.voorjaarsdagen.eu/proceedings/ category/52-companion-animal-scientificproceedings-

2011?download=1392:taylor_diagnosticapproach-to-exercise-intolerance-in-huntingdogs\&start=60.>. Accessed in: 07 jan. 2016. 
TILLEY, L.P. Essentials of canine and feline electrocardiography. Philadelphia: Lea \& Febiger, 1992. 470p.

TILLEY, L.P.; SMITH JR, F.W.K. Electrocardiography. In: TILLEY, L.P.; SMITH JR, F.W.K.; OYAMA, M.A.; SLEEPER, M.M. Manual of canine and feline cardiology. 4.ed. Philadelphia: WB Saunders, 2008. 443p.

VALBERG, S.J. Muscle anatomy, physiology and adaptations to exercise and training. In: HODGSON, D.R.; MCKEEVER, K.H.; MCGOWAN, C.M. The athletic horse. 2.ed. St. Louis: Saunders, 2014. p.174-201.
VITGER, A.D.; STALLKNECHT, B.M.; NIELSEN, D.H.; BJORNVAD, C.R. Integration of a physical training program in a weight loss plan for overweight pet dogs. J. Am. Vet. Med. Assoc., v.248, p.174-182, 2016.

YONEZAWA, L.A.; BARBOSA, T.S.; KOHAYAGAWA, A. Eletrocardiograma do equino. Rev. Ciênc. Agrovet., v.13, p.84-93, 2014.

YONEZAWA, L.A.; MACHADO, L.P.; SILVEIRA, V.F. et al. Exame eletrocardiográfico em equinos da raça Puro Sangue Árabe submetidos ao exercício em esteira de alta velocidade e à suplementação com vitamin E. Arch. Vet. Sci., v.14, p.134-142, 2009. 